\title{
Organisation du cytosquelette lors du développement précoce de la souris
}

Le cytosquelette (particulièrement les microtubules et les microfilaments) joue un rôle très important dans les toutes premières étapes du développement de l'embryon de souris. Il contribue à la mise en place de l'asymétrie cellulaire, conduisant à des divisions inégales en deux cellules filles dont le destin est différent. Ainsi en va-t-il de la seconde division méiotique de l'ovocyte, déclenchée par la fécondation et aboutissant à l'expulsion du second globule polaire, et de la polarisation des blastomères aux stades 8 et $\mathbf{l} 6$ cellules aboutissant à la différenciation entre masse cellulaire interne et trophectoderme, précurseurs respectivement de l'embryon et des annexes.

\section{Bernard Maro Joèl Aghion}

\section{ADRESSE}

B. Maro: Directeur de recherche au Cnrs. J. Aghion: Maître de conférences à l'Université Paris VII. Institut Jacques-Monod, Cnrs, université Paris VII, Inserm U. 257, Tour 43,

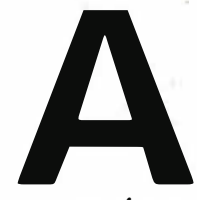

u cours du développement embryonnaire, des événements de détermination et de différenciation programmés par le génome conduisent, à partir des cellules initiales, à la formation des nombreuses lignées cellulaires spécialisées présentes dans l'organisme adulte. Classiquement, dans un certain nombre d'espèces (comme le nématode Caenorhabditis elegans), les lignages* cellulaires sont strictement définis et la disparition d'une cellule au cours du développement conduit à l'absence d'une partie de l'organisme adulte. Chez d'autres espèces (comme la souris), des phénomènes de compensation peuvent agir durant l'embryogenèse : des mécanismes de régulation cytoplasmique sont capables de moduler l'expression du programme génétique et donc de modifier le destin d'une cellule et de sa descendance.

\footnotetext{
* Lignage : néologisme pour "lineage ". Suc cession des cellules qui dérivent d'une ou plusieurs cellules et qui sont à l'origine d'un type cellulaire donné.
}

Récemment, ce type de mécanisme a, en fait, été également observé chez $C$. elegans [1]. Dans ces deux types d'organismes, l'organisation spatiale des cellules joue un rôle important dans les mécanismes de différenciation des cellules embryonnaires (tels que divisions cellulaires asymétriques ou localisation de déterminants cytoplasmiques). Enfin, il est indispensable que les diverses activités cellulaires soient intégrées, afin de permettre aux cellules de croître, de se diviser et d'interagir entre elles. Parmi les systèmes cytoplasmiques, le cytosquelette, et principalement le réseau de microtubules, pourrait assurer ce rôle de coordination [2, 3].

\section{Le cytosquelette}

Sous le terme cytosquelette, on désigne plusieurs réseaux de filaments protéiques présents dans le cytoplasme des cellules eucaryotes et impliqués dans l'architecture et la mobilité cellulaires [2]. Les deux principaux types de filaments composant le cytosquelette sont les microtubules et les microfilaments d'actine, tous deux constitués de pro- 


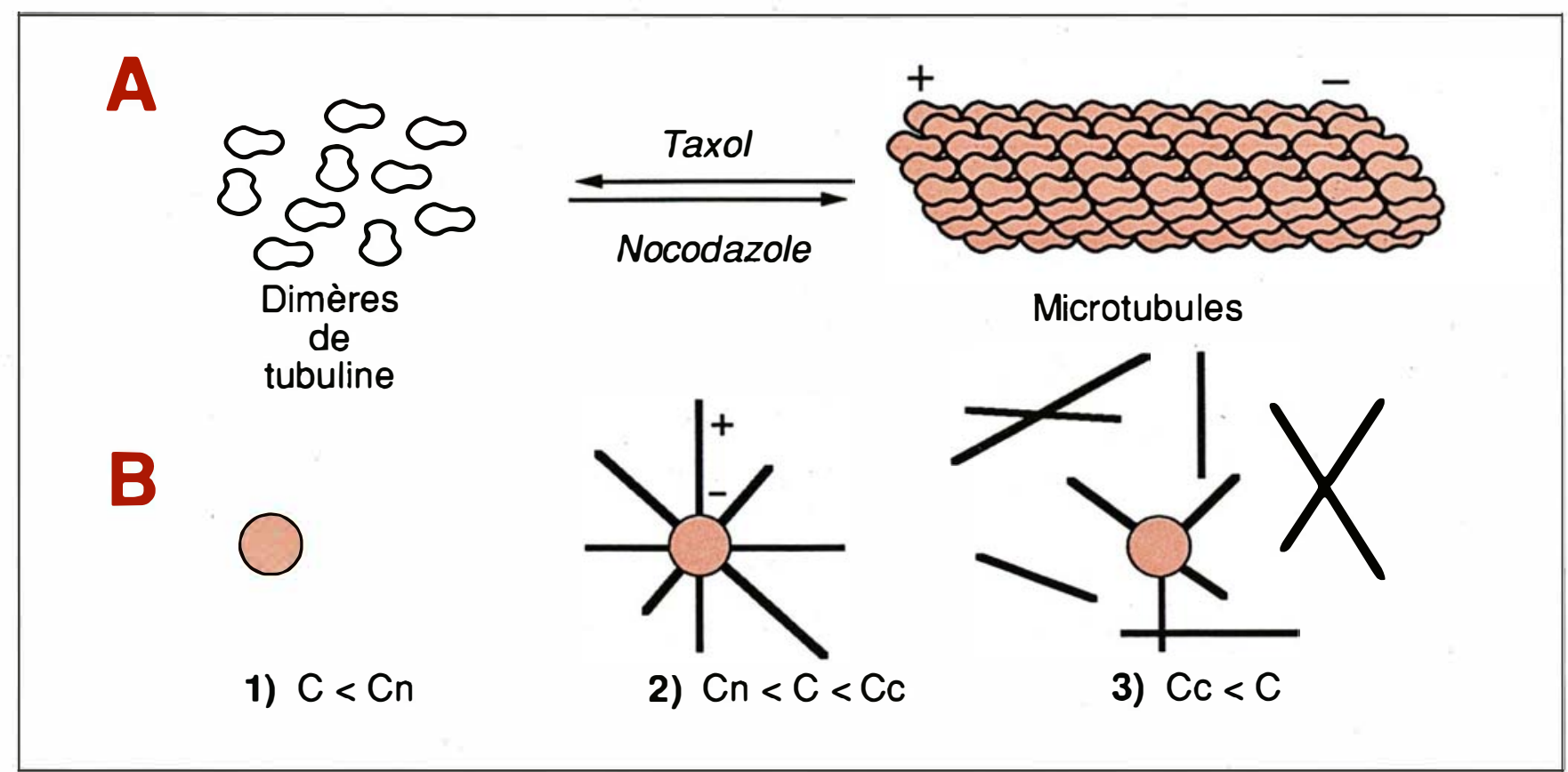

Figure 1. Microtubules et centres organisateurs des microtubules. A: les microtubules sont des tubes creux de $25 \mathrm{~nm}$ de diamètre formés de 13 protofilaments parallèles. Ce sont des polymères en équilibre dynamique avec la forme soluble, le dimère de tubuline $(\alpha-\beta)$. Le dimère de tubuline étant asymétrique, cela conduit à une asymétrie du polymère qui a deux extrémités ayant des propriétés cinétiques différentes, + et - . La croissance du microtubule est plus rapide à l'extrémité +. Le nocodazole se fixe au dimère de tubuline et bloque la polymérisation, alors que le taxol se fixe au microtubule et déplace l'équilibre vers le polymère et le stabilise. B : les centres organisateurs des microtubules (MTOCs) permettent aux microtubules de croître à une concentration de tubuline libre inférieure à la concentration critique pour la polymérisation de microtubules libres (nucléation). Les microtubules poussent toujours avec la même orientation à partir des MTOCs, l'extrémité étant toujours la plus proche du MTOC. 1. La concentration en tubuline (C) est inférieure à la concentration permettant la nucléation (Cn) des microtubules par les MTOCs (cercle gris). II n'y a pas de microtubules. 2. La concentration en tubuline (C) est comprise entre la concentration permettant la nucléation (Cn) des microtubules par les MTOCs et la concentration critique (Cc) permettant la formation de microtubules libres. Les microtubules poussent uniquement à partir des MTOCs. 3. La concentration en tubuline (C) est supérieure à la concentration critique (Cc) permettant la formation de microtubules libres.

téines polymérisées et dont les sousunités (tubuline et actine respectivement) peuvent s'associer et se dissocier rapidement. La troisième classe de filaments, les filaments intermédiaires, est composée de sous-unités formant des structures beaucoup plus stables.

Le réseau microtubulaire est constitué d'une part de structures fibrillaires, les microtubules - qui sont composés de tubuline et de protéines associées (MAPs, de l'anglais microtubule associated proteins) - , et d'autre part de structures individualisées d'où les microtubules semblent pousser, les centres organisateurs des microtubules (ou MTOCs, de l'anglais microtubule organising centers) (figure 1). Les centres organisateurs des microtubules des cellules ani$\mathrm{m} / \mathrm{s}^{\circ} 1$ vol. 5 , janvier 89 males, ou centrosomes, sont habituellement constitués par une paire de centrioles entourée par une structure dense aux électrons appelée matériel péricentriolaire. C'est ce matériel qui est responsable de l'activité biologique du MTOC, la nucléation des microtubules. Ce réseau est très dynamique et son organisation change au cours du cycle cellulaire. Dans une cellule en interphase, les microtubules forment un réseau dense, généralement organisé à partir du centrosome, alors qu'en mitose ils constituent les fibres du fuseau mitotique, un centrosome étant situé à chaque pôle. De plus, les microtubules contrôlent la localisation cellulaire de nombreux organites cytoplasmiques tels l'appareil de Golgi et les mitochondries. Enfin, dans plusieurs types cellulaires, il existe des sous-populations de microtubules plus stables qui contiennent de la tubuline modifiée (acétylée, détyrosinylée ${ }^{*}$ par des mécanismes agissant au niveau posttraductionnel.

Les microfilaments dont l'élément de base est l'actine, à laquelle de nombreuses protéines sont associées (telles que myosine, fodrine, villine, gelsoline...) forment un réseau pouvant être constitué de câbles cytoplasmiques, d'un feutrage sous-membranaire ainsi que de filaments individuels. Ils jouent un rôle dans la mobilité et la division cellulaires, ainsi que dans les mouvements intracellulaires.

** Perte de la tyrosine C-terminale de la sousunité a de tubuline. 


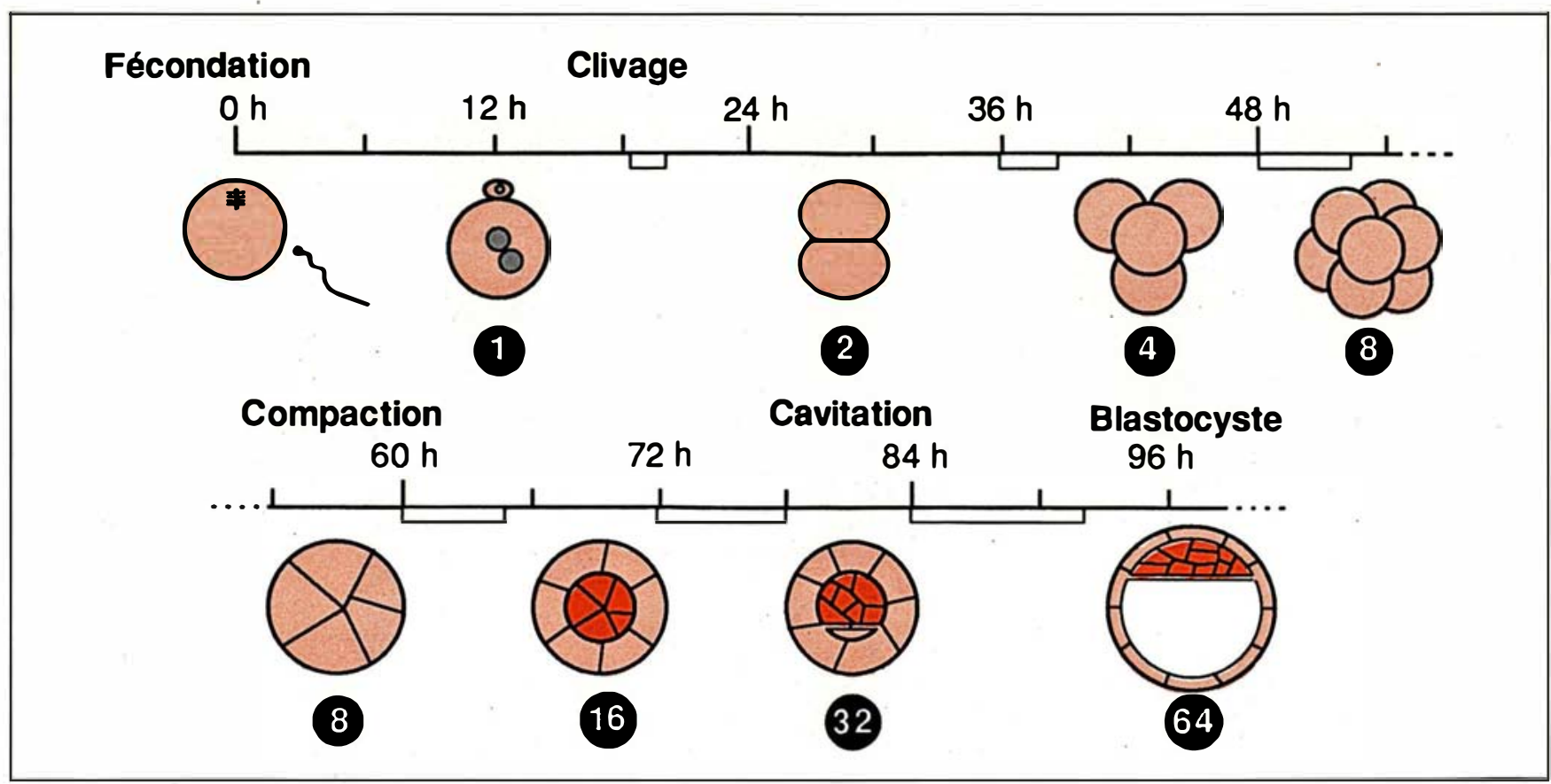

Figure 2. Développement de l'embryon de souris avant implantation. Le nombre de cellules présentes dans l'embryon à chaque stade est indiqué. A partir du stade 16, les cellules externes sont représentées avec un ombrage plus clair que les cellules internes. Sur l'échelle du temps, les zones en grisé représentent les périodes durant lesquelles une ou plusieurs cellules sont en mitose.

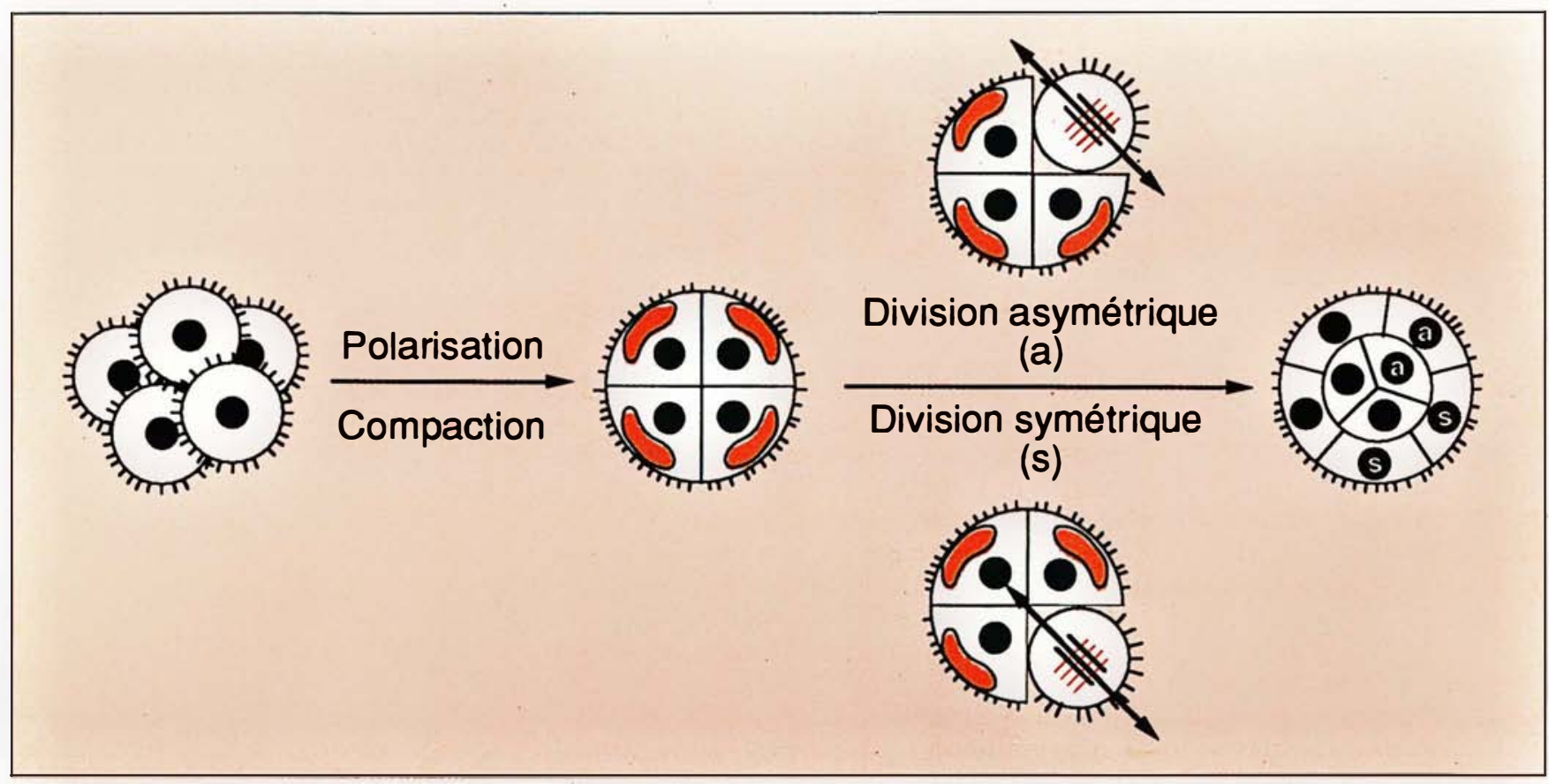

Figure 3. Mécanismes cytoplasmiques impliqués dans la divergence des deux premiers lignages de l'embryon. Polarisation : lors de la compaction, les blastomères, jusqu'alors symétriques, se polarisent (formation d'un pôle apical comprenant des microvillosités à la surface et divers organites dans le cytoplasme). Division asymétrique : lors de la division cellulaire, l'orientation du fuseau mitotique, parallèle ou orthogonal à l'axe de polarité de la cellule, détermine le phénotype des cellules filles: deux cellules polarisées si le fuseau est orthogonal (division symétrique) ou une cellule polarisée et une cellule non polarisée s'il est parallèle (division asymétrique). Seules les deux situations extrêmes sont représentées, le fuseau pouvant avoir n'importe quelle orientation par rapport à l'axe de polarité de la cellule. Adhésion : les cellules non polarisées sont adhésives sur toute leur surface alors que les cellules polarisées ne le sont que dans la région baso-latérale, la partie apicale n'étant pas adhésive. De ce fait, les cellules polarisées restent à l'extérieur de l'embryon et entourent les cellules non polarisées. 
Les filaments intermédiaires, qui, contrairement aux précédents sont exprimés avec une spécificité tissulaire, semblent jouer un rôle structural et mécanique dans l'organisation des tissus différenciés. Six types de filaments intermédiaires ont été décrits, incluant les filaments de kératine des cellules épithéliales.

Des interactions structurales et fonctionnelles ont été observées entre microfilaments et microtubules et entre filaments intermédiaires et microtubules [2]. En particulier, il a été montré que les microtubules semblaient contrôler dans certaines situations physiologiques l'activité des microfilaments.

\section{Le développement de la souris avant implantation}

Au moment de l'ovulation, l'œuf de souris est arrêté en deuxième métaphase de méiose (figure 2). La fécondation déclenche la reprise de la méiose et l'émission du second globule polaire. Les pronucléi mâle et femelle se forment et, après une phase de synthèse d'ADN, migrent au centre de l'œuf où les génomes maternel et paternel se mélangent pour la première fois en constituant la plaque métaphasique de première mitose. Au cours du deuxième cycle cellulaire, le génome embryonnaire est activé et les synthèses d'ARN commencent. Les deux premiers cycles cellulaires durent environ $18 \mathrm{~h}$ alors que les suivants durent environ $12 \mathrm{~h}$. Pendant cette période de clivage, les divisions cellulaires sont égales et asynchrones, sans aucune régularité dans l'orientation des plans de division. Au stade 8 cellules, l'embryon devient compact : les cellules s'aplatissent les unes sur les autres et les blastomères que l'on pouvait auparavant distinguer ne sont plus visibles individuellement. L'embryon, appelé morula, continue de se diviser et, au stade 16 cellules, on peut mettre en évidence pour la première fois un groupe de cellules internes, entièrement recouvert par une couche de cellules externes. Une accumulation de liquide, d'abord intracellulaire dans la partie basale des cellules externes, puis extracellulaire, débute au stade 32 et conduit à la formation d'une cavité appelée $\mathrm{m} / \mathrm{s} n^{\circ} l$ vol. 5 , jamier 89

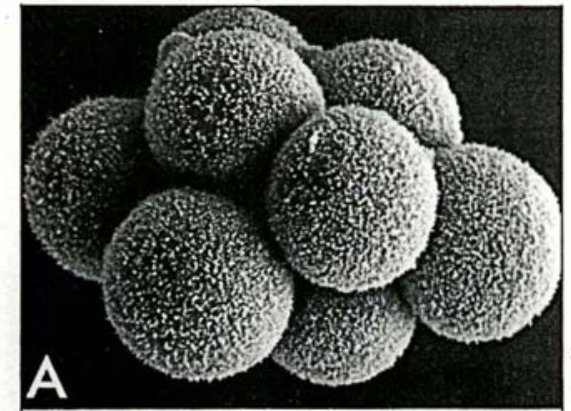

constituant l'embryon, influe sur leur différenciation: les cellules externes donnant préférentiellement naissance à des cellules du trophectoderme, les cellules internes à des cellules de la masse cellulaire interne $[5,6]$. Des différences morphologiques entre cellules internes et externes sont évidentes alors même que leur destin n'est pas fixé : les cellules externes sont polarisées, les cellules internes ne le sont pas [4].
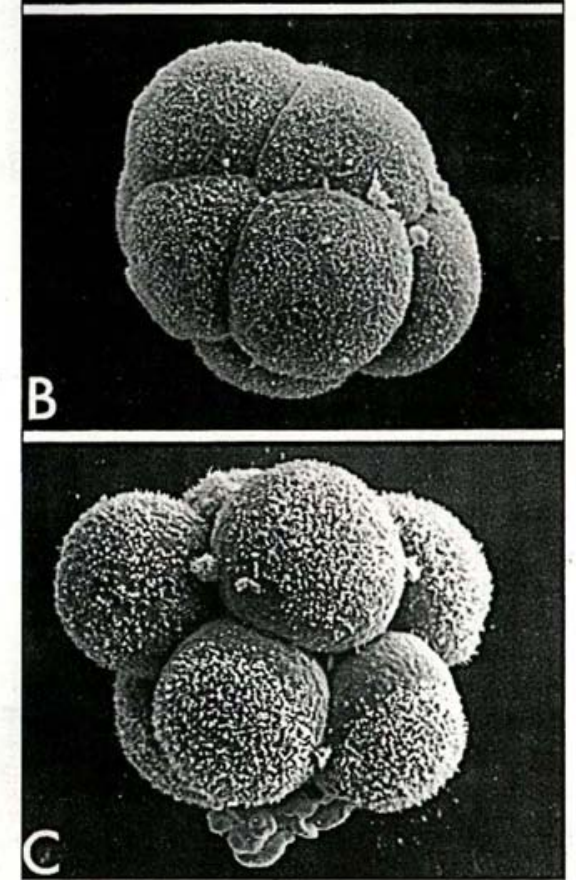

Figure 4. Embryons au stade $8 \mathrm{cel}$ lules observés par microscopie électronique à balayage. A : Embryon non compacté; noter que les blastomères sont bien individualisés et qu'ils sont couverts de microvillosités. $\boldsymbol{B}$ : Embryon compacté. C : Embryon compacté incubé dans un milieu appauvri en calcium pour inhiber l'adhésion intercellulaire; noter la présence de pôles de microvillosités à la surface des blastomères.

le blastocœele. De l'accroissement de taille de cette cavité résulte la position excentrée de la masse cellulaire interne. Dans le blastocyste, un épithélium qui dérive des cellules externes, le trophectoderme, entoure, d'une part, le blastocœele et, d'autre part, la masse cellulaire interne. Le trophectoderme est à l'origine des tissus des annexes embryonnaires alors que les cellules de la masse cellulaire interne sont à l'origine de l'embryon lui-même [4].

La position des cellules, à l'intérieur ou à l'extérieur de l'amas cellulaire
C'est durant la compaction qu'apparaissent les premières cellules polarisées. Au début du stade 8 cellules, les blastomères sont radialement symétriques et bien individualisés: des microvillosités couvrent toute leur surface, les organites cytoplasmiques sont distribués de manière homogène dans tout le cytoplasme et il n'existe pas encore de communication intercellulaire (figures 3 et 4). Les premières modifications observables sont, d'une part, la formation de jonctions communicantes et, d'autre part, la formation d'un pôle apical cytoplasmique constitué par divers organites tels les endosomes ou les microfilaments [4]. Puis, les cellules s'aplatissent les unes sur les autres (compaction) par un mécanisme lié à une molécule d'adhésion cellulaire, l'uvomoruline [7]. Les microvillosités disparaissent des zones de contact intercellulaires ainsi que des régions baso-latérales, ce qui restreint au pôle apical l'existence de microvillosités [8]

Ce phénomène de polarisation qui se produit lors de la compaction est un élément clé de la différenciation cellulaire conduisant aux deux populations cellulaires présentes dans le blastocyste. En effet, l'orientation de l'axe du fuseau mitotique par rapport à l'axe de polarité de la cellule détermine le phénotype des cellules filles au stade 16 cellules, polarisées (présumées être à l'origine des cellules $\mathrm{du}$ trophectoderme) ou non (présumées être à l'origine des cellules de la masse cellulaire interne) : s'ils sont parallèles, une cellule fille sera polarisée l'autre non, s'ils sont perpendiculaires, elles seront toutes deux polarisées (figure 3) [9]. Il faut noter que seules les deux situations extrêmes ont été décrites, le fuseau pouvant avoir n'importe quelle orientation par rapport à l'axe de polarité de la cellule à ce 
stade du développement [10]. Ce phénomène de polarisation est à l'origine d'un autre mécanisme qui joue un rôle important dans la différenciation du blastocyste: l'adhésion intercellulaire. En effet, la membrane plasmique des cellules polarisées est constituée de deux domaines qui ont des propriétés adhésives différentes: le domaine basolatéral est adhésif et le domaine apical non adhésif. La surface des cellules non polarisées étant adhésive, ceci conduit à l'encerclement des cellules non polarisées par les cellules polarisées [4].

Il est possible d'isoler les deux types de cellules et d'étudier leur évolution dans différentes conditions expérimentales. Lorsqu'une cellule interne est placée à l'extérieur de l'embryon

\section{RÉFÉRENCES}

1. Priess JR, Thomson JN. Cellular interactions in early Caenorhabditis elegans embryos. Cell 1987; 48 : 214-50.

2. Schliwa M. The cytoskeleton : an introductory survey. New York : Springer Verlag, 1986.

3. Kirschner M, Mitchison T. Beyond selfassembly: from microtubules to morphogenesis. Cell 1986 ; 45 : 329-42.

4. Johnson MH, Maro B. Time and space in the early mouse embryo: a cell biological approach to cell diversification. In : Rossant J, Pedersen R, eds. Experimental Approaches to Mammalian Embryonic Development. Cambridge: Cambridge University Press, 1986 : 35-65.

5. Tarkowski AK, Wroblewska J. Development of blastomeres of mouse eggs isolated at the 4- and 8-cell stage. J Embryol Exp Morph 1967; 18 : 1155-80.

6. Fleming TP. A quantitative analysis of cell allocation to trophectoderm and inner cell mass in the mouse blastocyst. Dev Biol 1987 ; 119: 520-31.

7. Hyafil. F, Morello D, Babinet C, Jacob F. A cell surface glycoprotein involved in the compaction of embryonal carcinoma cells and cleavage stage embryos. Cell 1980; 21 : 927-34.

8. Ducibella $T$, Ukena $T$, Karnovsky M, Anderson E. Changes in cell surface and cortical cytoplasmic organisation during early embryogenesis in the preimplantation mouse embryo. J Cell Biol $1977 ; 74$ : 153-67.

9. Johnson MH, Ziomek CA. The foundation of two distinct cell lineages within the mouse morula. Cell $1981 ; 24: 71-80$

10. Pickering SJ, Maro B, Johnson $\mathrm{MH}$, Skepper J. The influence of cell contact on the division of mouse 8-cell blastomeres.

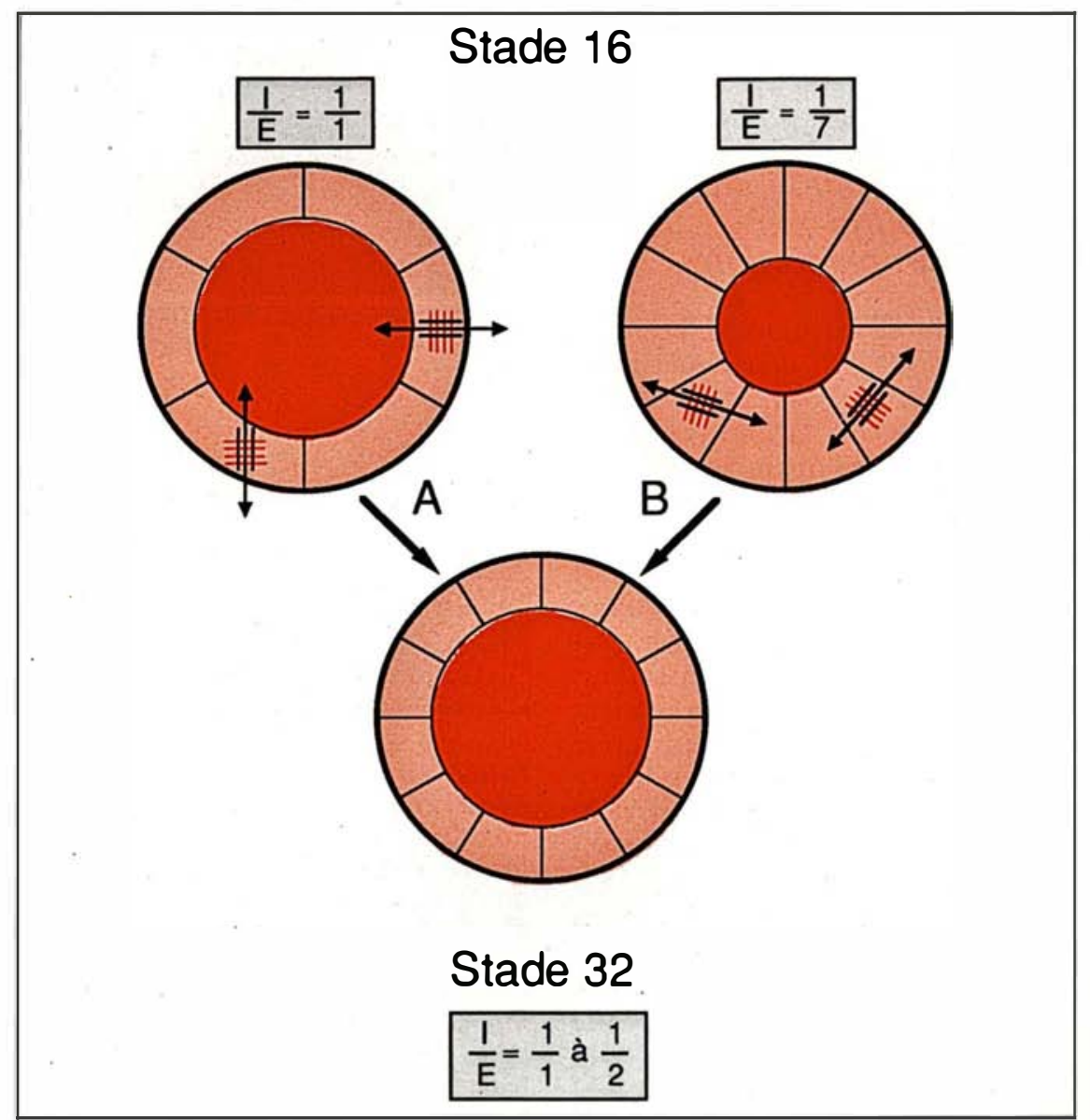

Figure 5. Régulation du rapport entre le nombre de cellules internes et externes durant la transition du stade 16 au stade 32 cellules. A : si le nombre de cellules internes est grand (rapport cellules internes/cellules externes $=1 / 1$ ), les cellules externes seront aplaties et les fuseaux mitotiques s'orienteront parallèlement à la surface. Dans ces conditions les divisions seront symétriques et ne donneront que des cellules filles externes (polarisées). Les cellules internes se divisant symétriquement, le rapport cellules internes/cellules externes (I/E) sera maintenu. $B$ : si le nombre de cellules internes est faible $(I / E=1 / 7)$, les cellules externes seront pyramidales et les fuseaux mitotiques s'orienteront perpendiculairement à la surface. Dans ces conditions, les divisions seront asymétriques et donneront des cellules filles externes (polarisées) et internes (non polarisées). Le rapport I/E augmentera fortement et tendra vers 1/1. Seules les deux situations extrêmes sont représentées, dans la situation intermédiaire (I/E $=1 / 4$ à $1 / 3)$ les cellules seront plus cuboïdales et le fuseau pourra avoir n'importe quelle orientation par rapport à l'axe de polarité de la cellule. Dans ces conditions les divisions seront asymétriques ou symétriques et donneront plus de cellules filles externes (polarisées) qu'internes (non polarisées). Le rapport I/E augmentera légèrement. 


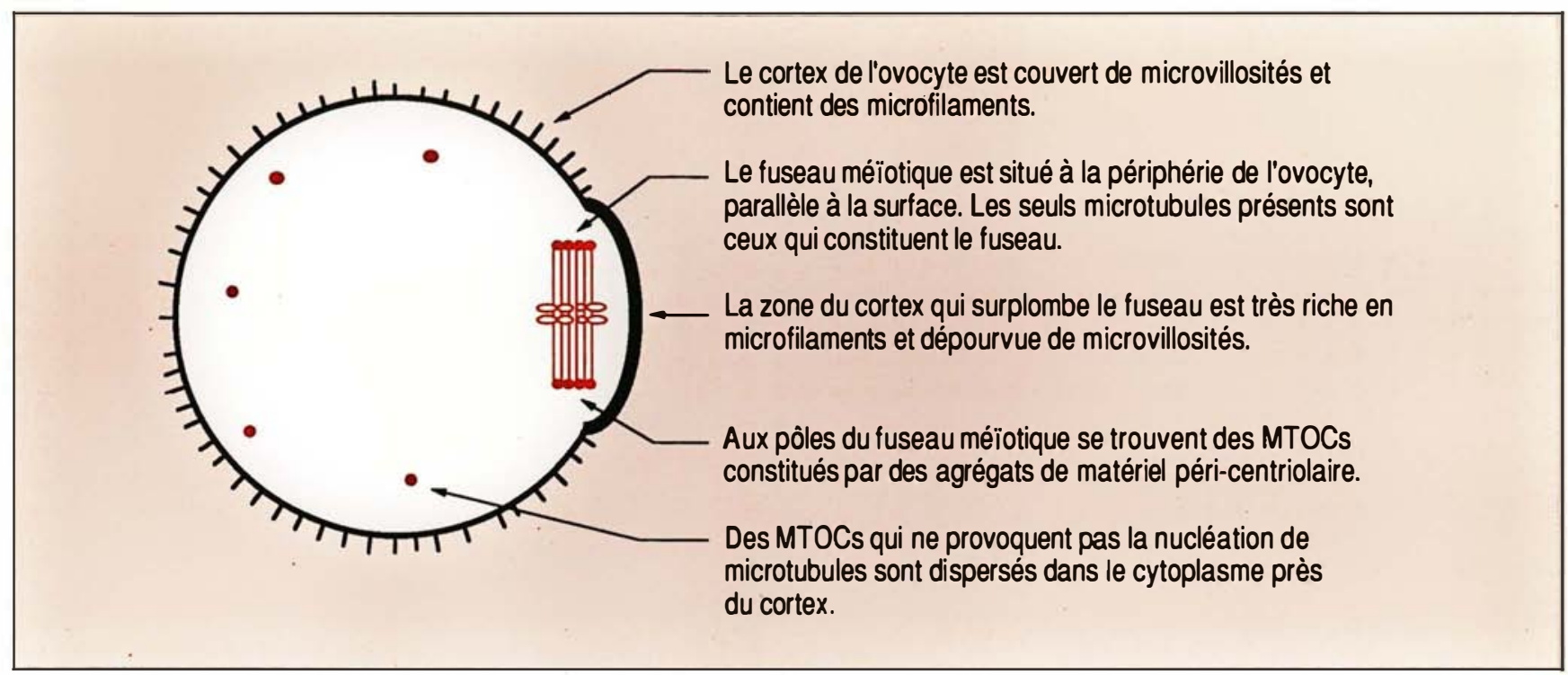

Figure 6. Organisation du cytosquelette de l'ovocyte de souris.

et n'est plus entourée complètement, son phénotype évolue progressive vers celui d'une cellule externe (polarisée) et elle sera à l'origine de cellules du trophectoderme. Les cellules externes quant à elles peuvent avoir des descendantes internes non polarisées dans la masse cellulaire interne [6], La conséquence de divisions cellulaires asymétriques.

Au stade 16 cellules, le nombre de cellules internes varie de 2 à 7 et le nombre de cellules externes de 14 à 9 soit un rapport de $1 / 7$ à environ 1/1 [6]. Cette variabilité s'estompe au stade 32 cellules, où le rapport est compris entre $1 / 2$ et $1 / 1[6]$. Cette compensation s'effectue par un mécanisme d'orientation préférentiel de l'axe du fuseau de division des cellules externes qui est fonction de la forme de ces cellulles. Le volume de l'embryon étant constant, plus le nombre de cellules internes est grand, plus les cellules externes sont aplaties. Ceci favorise une orientation des fuseaux mitotiques parallèle à la surface de l'embryon et donc des divisions symétriques qui ne donnent que des cellules externes (figure $5 A$ ). Les cellules internes se divisant symétriquement, la proportion de cellulles externes ne changera donc pas. $\mathrm{Si}$, en revanche, le nombre de cellules internes est faible, les cellules externes seront pyramidales, les $\mathrm{m} / \mathrm{s} \mathrm{n}^{\circ} 1$ vol. 5 , janvier 89 fuseaux perpendiculaires à la surface de l'embryon (figure $5 B$ ) et les divisions asymétriques. Ceci entraîne une augmentation de la proportion de cellules internes présentes dans l'embryon au stade suivant.

\section{Rôle du cytosquelette lors du développement}

Ovocyte et fécondation. Les événements qui prennent place après la fusion entre l'ovocyte et le spermatozoïde, tels que l'extrusion du deuxième globule polaire, la formation et la migration des pronucléi, le mélange des chromosomes maternels et paternels, sont d'une importance capitale pour l'établissement et l'activation du génome embryonnaire. En effet tout dysfonctionnement à ce stade peut conduire à la perte de l'embryon ou à des malformations congénitales. La compréhension de ces mécanismes nécessite l'étude de l'organisation intracellulaire ainsi que celle de la mobilité cellulaire, processus sous la dépendance des éléments du cytosquelette (microtubules et microfilaments). L'organisation du cytosquelette de l'ovocyte est détaillée dans la figure 6 .

Dans l'ovocyte, le fuseau métaphasique de seconde division de méiose est situé près du cortex, parallèle à celuici (figure $7 A$ ). Il existe deux domaines corticaux distincts dans l'ovocyte dont un très riche en microfilaments et dépourvu de microvillosités au voisinage du fuseau méiotique (figure $7 B$ ). Après fécondation, le second globule polaire se forme dans ce domaine et une autre région riche en microfilaments et dépourvue de microvillosités apparaît dans le cortex situé au-dessus de la tête du spermatozoïde (figure 7C). Ces différenciations locales du cortex disparaissent lors de la formation des pronucléi [11].

Les seuls microtubules présents dans l'ovocyte sont ceux qui constituent les fibres fusoriales. Bien que le fuseau méiotique soit stable pendant plusieurs heures (jusqu'au moment de la fécondation), les microtubules qui le composent polymérisent et dépolymérisent très rapidement, leur dynamique étant équivalente à celle des microtubules du fuseau mitotique [12]. Il n'y a pas de centrioles dans l'ovocyte de souris, un agrégat de matériel dense aux électrons correspondant au matériel péricentriolaire de la plupart des cellules somatiques animales se trouve à chaque pôle du fuseau méiotique[13]. Le spermatozoïde n'apporte pas de centriole fonctionnel lors de la fécondation, et les centrioles apparaissent tardivement dans l'embryon (ils sont présents au stade blastocyste). De nombreux agrégats de matériel péri- 


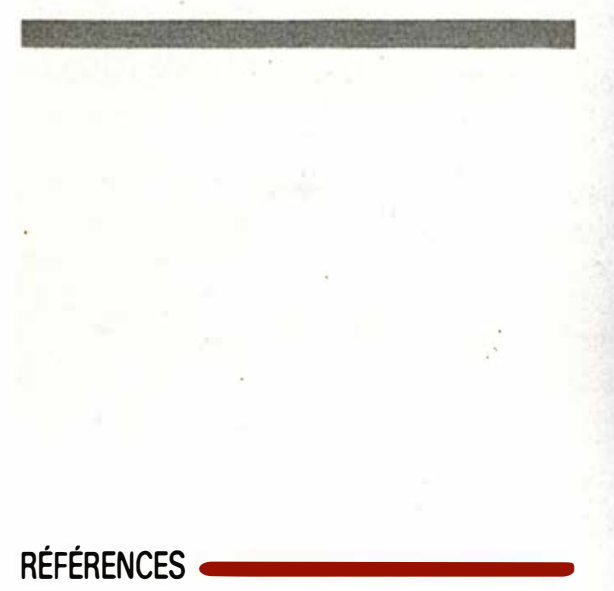

11. Maro B, Johnson MH, Webb M, Flach G. Mechanism of polar body formation in the mouse oocyte: an interaction between the chromosomes, the cytoskeleton and the plasma membrane. J Embryol Exp Morph $1986 ; 92: 11-32$.

12. De Pennart $H$, Houliston $H$, Maro $B$ Post-translational modifications of tubulin and the dynamics of microtubules in mouse oocytes and zygotes. Biol Cell 1988 (soumis pour publication).

13. Szollosi D, Calarco P, Donahue RP. Absence of centrioles in the first and second meiotic spindles of mouse oocytes. J Cell Sci $1972 ; 11: 521-41$.

14. Maro B, Howlett SK, Webb M. Nonspindle microtubule organizing centers in metaphase II-arrested mouse oocytes. J Cell Biol 1985 ; 101 : 1665-72.

15. Schatten G, Simerly C, Schatten H Microtubule configuration during fertilization, mitosis and early development in the mouse. Proc Natl Acad Sci USA 1985; 82 : 4152-6.

16. Maro B, Johnson $\mathrm{MH}$, Pickering SJ Flach G. Changes in the actin distribution during fertilization of the mouse egg. I Embryol Exp Morph $1984 ; 81$ : 211-37.

17. Houliston E, Pickering SJ, Maro B Redistribution of microtubules and peri-centriolar material during compaction in mouse blastomeres. J Cell Biol 1987 ; 104 : 1299-308.

18. Houliston H, Maro B. Post-translational modification of distinct microtubule subpopulations during cell polarization and differentiation in the mouse preimplantation embryo. J Cell Biol 1989 (sous presse).

19. Fleming TP, Cannon P, Pickering SJ. The role of the cytoskeleton in generating and stabilising an asymmetric distribution of endocytotic organelles during the process of cell polarization in the mouse preimplantation embryo. Dev Biol 1986; 113 : 406-19.

20. Goodall H, Maro B. Major loss of junctional communication during mitosis in early mouse embryos. J Cell Biol 1986 ; 102 :

Figure 7. Mise en évidence de la tubuline ot de l'actine dans des ovocytes par immunofluorescence indirecte. A : Ovocyte marqué par un anticorps anti-tubuline (cliché de gauche). Les chromosomes sont marqués par le Hoechst 33258 (cliché de droite). La flèche indique la position des chromosomes. B : Ovocyte marqué par un anticorps anti-actine (cliché de gauche). Les chromosomes sont marqués par le Hoesch 33258 (cliché de droite). La flèche indique la position des chromosomes. C: Ovocyte fécondé expulsant le deuxième globule polaire (flèche) marqué par un anticorps antiactine (cliché de gauche). Les chromosomes sont marqués par le Hoescht 33258 (cliché de droite). Noter l'importance du marquage du cortex en regard de la tête du spermatozoïde (petite flèche).

centriolaire qui ne sont pas liés aux pôles du fuseau méiotique sont présents dans l'ovocyte près de la membrane plasmique [14]. Après fécondation et entrée en interphase, ces MTOCs entraînent la nucléation des microtubules du réseau interphasique [15]. Ils sont ensuite impliqués dans le mélange des deux groupes haploïdes de chromosomes et se retrouvent aux pôles du fuseau mitotique de première division. Grâce à l'utilisation d'inhibiteurs spécifiques, il est possible de montrer que les microtubules et les microfilaments jouent un rôle important dans la migration des pronucléi vers le centre de l'œuf.

Lorsque les ovocytes sont préincubés en présence de nocodazole, drogue qui induit la dépolymérisation des microtubules, la transition entre métaphase de méiose et interphase ne se produit pas, bien que la tête du spermatozoïde ait pénétré à l'intérieur de l'œuf. De plus, la plaque métaphasique se désagrège et les chromosomes se dispersent en petits groupes qui restent localisés près de la membrane plasmique [16]. Dans ces conditions, on peut observer une région de la surface cellulaire riche en microfilaments et dépourvue de microvillosités en regard de chacun des groupes de chromosomes. Quand, après dispersion des chromosomes par le nocodazole, on enlève la drogue du milieu de culture, on observe tout d'abord une polymérisa- tion de tubuline autour de chacun des groupes de chromosomes, puis le recrutement d'agrégats de matériel péricentriolaire qui vont former les pôles de fuseaux multiples (jusqu'à 4-5) [16]. Enfin, la deuxième division de méiose se termine et plusieurs globules polaires sont expulsés d'une même cellule. Dans l'ovocyte de souris, les chromosomes sont donc capables de modifier l'organisation du cytosquelette (microfilaments et microtubules) dans leur environnement, ce qui permet la division inégale qui donne naissance au globule polaire et à l'embryon [16]. Cette division est liée à l'existence d'un domaine particulier du cortex dans lequel le sillon de division est confiné.

Compaction et mise en place de la polarité cellulaire. Lors de la compaction se produisent les événements cellulaires impliqués dans la divergence des premiers types cellulaires et conduisant à la formation de la masse cellulaire interne et du trophectoderme (figure 3).

Une réorganisation du réseau microtubulaire se produit lors de la mise en place de la polarité cellulaire : la majeure partie des microtubules disparaît rapidement des zones de contact intercellulaires et se concentre dans la partie apicale de la cellule. Cette redistribution des microtubules précède la redistribution massive du matériel péricentriolaire 
dans la partie apicale de la cellule [17]. Il faut noter qu'une souspopulation de microtubules plus stables et acétylés est localisée près des zones de contacts intercellulaires [18]. Le rôle des microtubules dans le processus de compaction a été étudié à l'aide de drogues interférant avec les microtubules : le nocodazole, qui induit la dépolymérisation des microtubules, et le taxol, qui induit la formation d'un réseau non organisé de microtubules très stables (figure 1). Les effets des drogues peuvent être résumés ainsi [17]: (1) la polarité cytoplasmique dépend du réseau microtubulaire [19];(2) la formation des jonctions communicantes ( $g a p$ ) est inhibée par le taxol alors que le nocodazole est sans effet [20], ce qui suggère un rôle inhibiteur des microtubules; (3) l'aplatissement des cellules les unes sur les autres est inhibé par le taxol alors que le nocodazole accélère ce processus [21], ce qui suggère que les microtubules inhibent également ce phénomène ; (4) enfin, les microtubules jouent un rôle dans le développement de la polarité de surface. Les microvillosités sont localisées sur toute la partie exposée de la surface des blastomères après traitement par le nocodazole ou par le taxol [21]. Cependant, en l'absence d'adhésion intercellulaire, les microtubules sont absolument nécessaires à la mise en place de ce pôle de microvillosités [22].

Lorsque l'on confronte les résultats obtenus concernant, d'une part, la redistribution du réseau microtubulaire lors de la compaction et, d'autre part, l'effet des drogues interférant avec les microtubules pendant la même période, il est possible de proposer un modèle mettant en évidence le rôle de ce réseau. La disparition des microtubules les plus dynamiques dans la partie basale de la cellule faciliterait la disparition des microvillosités et favoriserait l'adhésion intercellulaire et la formation des jonctions communicantes, alors que leur présence dans la partie apicale stabiliserait le pôle de microvillosités et celui constitué par les organites cytoplasmiques. Finalement, la localisation du matériel péricentriolaire dans la partie apicale de la cellule renforcerait l'asymétrie initiale existant dans la distribution des microtubules, en permettant la nucléation de $\mathrm{m} / \mathrm{s} n^{\circ} 1$ vol. 5 , janvier 89 nouveaux microtubules. Il semble donc que les microtubules jouent un rôle important dans la coordination des divers changements de l'organisation cellulaire qui prennent place lors de la compaction (polarisation), leur rôle devenant essentiel en l'absence d'adhésion intercellulaire [17, 18].

Durant la compaction, des structures riches en actine ont été localisées par immunofluorescence au niveau du pôle de microvillosités ainsi que dans la région du pôle cytoplasmique. $\mathrm{La}$ cytochalasine $\mathrm{D}$, qui désorganise des microfilaments, inhibe l'aplatissement des cellules les unes sur les autres ainsi que la formation des pôles cytoplasmiques sans toutefois pouvoir disperser ceux qui sont déjà formés. Elle ne bloque pas l'apparition du pôle de surface, mais modifie son organisation [4, 23]. Il semble donc que, lors de la compaction, les microfilaments soient impliqués dans les événements terminaux tels que le mouvement de certains organites cytoplasmiques et l'aplatissement des cellules plutôt que dans la coordination de ces événements.

Divisions asymétriques et divergence des premiers « lignages». Lors de la mitose qui suit la compaction (transition du stade 8 cellules au stade 16 cellules) et qui joue un rôle clé pour la divergence des deux types cellulaires présents dans la morula, seule la polarité de surface est maintenue : en effet les blastomères s'arrondissent, les organites du pôle cytoplasmique se dispersent et les jonctions communicantes ne sont plus fonctionnelles [20, 24]. Il est possible d'abolir cette polarité de surface par un arrêt de plusieurs heures en mitose (induit par le nocodazole) : le pôle de microvillosités augmente de taille jusqu'à couvrir toute la surface de la cellule. Après avoir levé le blocage en supprimant la drogue : le pourcentage de divisions symétriques s'accrô̂t. Néanmoins, la stabilité du pôle de surface est bien supérieure à la durée normale de la mitose, ce qui permet de conserver cette asymétrie lors de la division [25]. Au stade 16, en interphase, les jonctions sont de nouveau fonctionnelles, les cellules s'aplatissent de nouveau et la polarité cytoplasmique se reforme dans les cellules polarisées à leur surface [20, 24].

Il est possible de mettre en évidence une différence dans l'organisation des microtubules entre cellules polarisées et non polarisées aux stades 16 et 32 cellules : le cortex des cellules internes est enrichi en microtubules acétylés plus stables [18]. La question reste de savoir si cette population de microtubules acétylés joue un rôle dans les mécanismes de différenciation des cellules internes.

Les filaments intermédiaires, troisième réseau du cytosquelette, ont souvent été décrits comme étant des marqueurs de différenciation. Les filaments intermédiaires de kératine sont les seuls filaments mis en évidence de manière certaine dans l'embryon précoce de souris. En effet, certaines cytokératines semblent caractéristiques du trophectoderme [26, 27]. Cependant, les synthèses de protéines $[28,29]$ et d'ARNm [30] peuvent être détectées avant le stade blastocyste. Les premiers filaments apparaissent au stade 8 , dans quelques cellules. Au stade 16 , ils sont détectés dans un nombre croissant de cellules autant externes qu'internes. Au stade 32, puis dans les blastocystes, toutes les cellules externes contiennent de nombreux filaments, alors que les cellules internes perdent progressivement ces filaments [29]. Ces résultats suggèrent que des mécanismes liés aux interactions cellulaires sont impliqués dans la régulation de l'expression génétique des cytokératines, qui est maintenue dans les cellules externes qui formeront le trophectoderme.

\section{Conclusion}

Dans l'embryon précoce de souris, des mécanismes de régulation cytoplasmiques contrôlent les premières étapes du développement. Ces mécanismes épigénétiques ont un rôle primordial dans ce système, à la différence de ce qui semble se passer dans d'autres systèmes. Le cytosquelette en est un élément important, en particulier lors de la mise en place des asymétries qui permettront la naissance de deux types cellulaires distincts après une division inégale, à la fois lors de l'expulsion du deuxième globule polaire (différenciation terminale de l'ovocyte) et lors de la diver$\mathrm{g}$ en c e d e s d e u x p r e mi ères populations cellulaires après compaction de l'embryon 


\section{RÉFÉRENCES}

21. Maro B, Pickering SJ. Microtubules influence compaction in preimplantation mouse embryos. J Embryol Exp Morph 1984 ; 84 : 217-32.

22. Maro B, Houliston E, de Pennart $\mathbf{H}$ Microtubule dynamics and cell diversification in the mouse preimplantation embryo Protoplasma 1988 ; 145 : 160-6.

23. Fleming TP, Pickering SJ, Qasim F Maro $B$. The generation of cell surface pola rity in mouse 8-cell blastomeres: the role of cortical microfilaments analysed using cytochalasin D. J Embryol Exp Morph 1986 ; 95 : 169-91.

24. Maro B, Johnson MH, Pickering SJ, Louvard D. Changes in the distribution of membranous organelles during mouse early embryogenesis. J Embryol Exp Morph 1985 $90: 287-309$

25. Johnson $\mathrm{MH}$, Pickering SJ, Dhiman A, Radcliffe GS, Maro B. Cytocortical organisation during natural and prolonged mitosis of mouse 8-cell blastomeres. Development 1988 ; 102 : 143-58

26. Brûlet P, Babinet C, Kemler R, Jacob F Monoclonal antibodies against trophectodermal-specific markers during mouse blastocyst formation. Proc Natl Acad Sci USA 1980 ; 77 : 4113-7.

27. Jackson BW, Grund C, Schmid E Burki K, Franke WW, Illmensee K. Formation of cytoskeletal elements during mouse embry'ogenesis. Intermediate filaments of the cytokeratin type and desmosomes in preimplantation embryos. Differentiation $1980 ; 17$ : 161-79.

28. Oshima RG, Howe WE, Klier FG, Adamson ED, Shevinsky LH. Intermediate filament protein synthesis in preimplantation murine embryos. Dev Biol 1983 ; 99 : 447-55.

29. Chisholm JC, Houliston E. Cytokeratin assembly in the mouse preimplantation embryo. Development 1987 ; 101 : 565-82.

30. Duprey P, Morello D, Vasseur M, et al. Expression of the cytokeratin endo A gene during early mouse embryogenesis. Proc Natl

\section{Summary}

In this paper, we review the role of microtubules and microfilaments during mouse preimplantation development. These cytoskeletal elements are involved in many cellular functions such as cell organisation and cell division, all of these being of importance for the cellular mechanisms that play a major role in the formation of the blastocyst. The setting up of cell polarity at compaction and the mechanisms underlying asymmetrical cell divisions (during polar body formation and cell diversification after compaction) are discussed.
TIRÉS A PART

B. Maro. 